\title{
Corrigendum: Transcriptome Analysis of Maize Immature Embryos Reveals the Roles of Cysteine in Improving Agrobacterium Infection Efficiency
}

\author{
Yan Liu ${ }^{1,2}$, Zhiqiang Zhang ${ }^{1,2}$, Junjie $\mathrm{Fu}^{2}$, Guoying Wang ${ }^{2}$, Jianhua Wang ${ }^{1 *}$ and \\ Yunjun Liu $^{2 *}$ \\ ${ }^{1}$ College of Agronomy and Biotechnology, China Agricultural University, Beijing, China, ${ }^{2}$ Institute of Crop Sciences, Chinese \\ Academy of Agricultural Sciences, Beijing, China
}

Keywords: Agrobacterium, infection efficiency, cysteine, maize embryo, transcriptome

\section{OPEN ACCESS}

Edited and reviewed by:

Paulo Arruda

Universidade Estadual de Campinas,

Brazil

*Correspondence: Jianhua Wang

wangjh63@cau.edu.cn

Yunjun Liu

liuyunjun@caas.ch

Specialty section: This article was submitted to

Plant Biotechnology,

a section of the journal

Frontiers in Plant Science

Received: 24 October 2017

Accepted: 31 October 2017

Published: 07 November 2017

Citation:

Liu Y, Zhang Z, Fu J, Wang G, Wang J and Liu Y (2017) Corrigendum:

Transcriptome Analysis of Maize Immature Embryos Reveals the Roles of Cysteine in Improving

Agrobacterium Infection Efficiency.

Front. Plant Sci. 8:1964.

doi: 10.3389/fp/s.2017.01964

\section{A corrigendum on}

Transcriptome Analysis of Maize Immature Embryos Reveals the Roles of Cysteine in Improving Agrobacterium Infection Efficiency

by Liu, Y., Zhang, Z., Fu, J., Wang, G., Wang, J., and Liu, Y. (2017). Front. Plant Sci. 8:1778.

doi: $10.3389 / f p l s .2017 .01778$

In the original article, there was a mistake in Table 3 as published. In original Table 3, four aquaporin genes (GRMZM2G041980, GRMZM2G392975, GRMZM2G126582, GRMZM2G081843) were repeated two times, and we neglected to include several upregulated glycosyltransferase genes (GRMZM2G120016, GRMZM5G834303). The corrected Table 3 appears below.

In the original article, there was an error. The reads from the HiII embryo samples were incorrectly presented as " $7,432,161-15,904,122$ ". The correct reads from the HiII embryo samples should be "7,432,161-15,881,554" as shown in Table 1 .

A correction has been made to (Results), (Maize Embryo Transcriptome Profiling), (Paragraph 1):

To investigate the mechanism of cysteine-related transformation efficiency improvement, we performed transcriptome analysis of the maize embryos cultured on the medium with or without cysteine. The experiment was performed with four independent biological replicates. We obtained $7,432,161-15,881,554$ reads from the HiII embryo samples and more than $78.73 \%$ of the reads were mapped to the B73 reference genome. We obtained 20,119,176-24,789,278 reads from the inbred line Z31 embryo samples and $73.66 \%$ of the reads were mapped to the B73 reference genome (Table 1). Maize has $\sim 30,000$ genes and the sequencing depth we acquired was enough for subsequent analysis. The authors apologize for this error and state that this does not change the scientific conclusions of the article in any way. 
TABLE 3 | DEGs which involved in the cell wall metabolism.

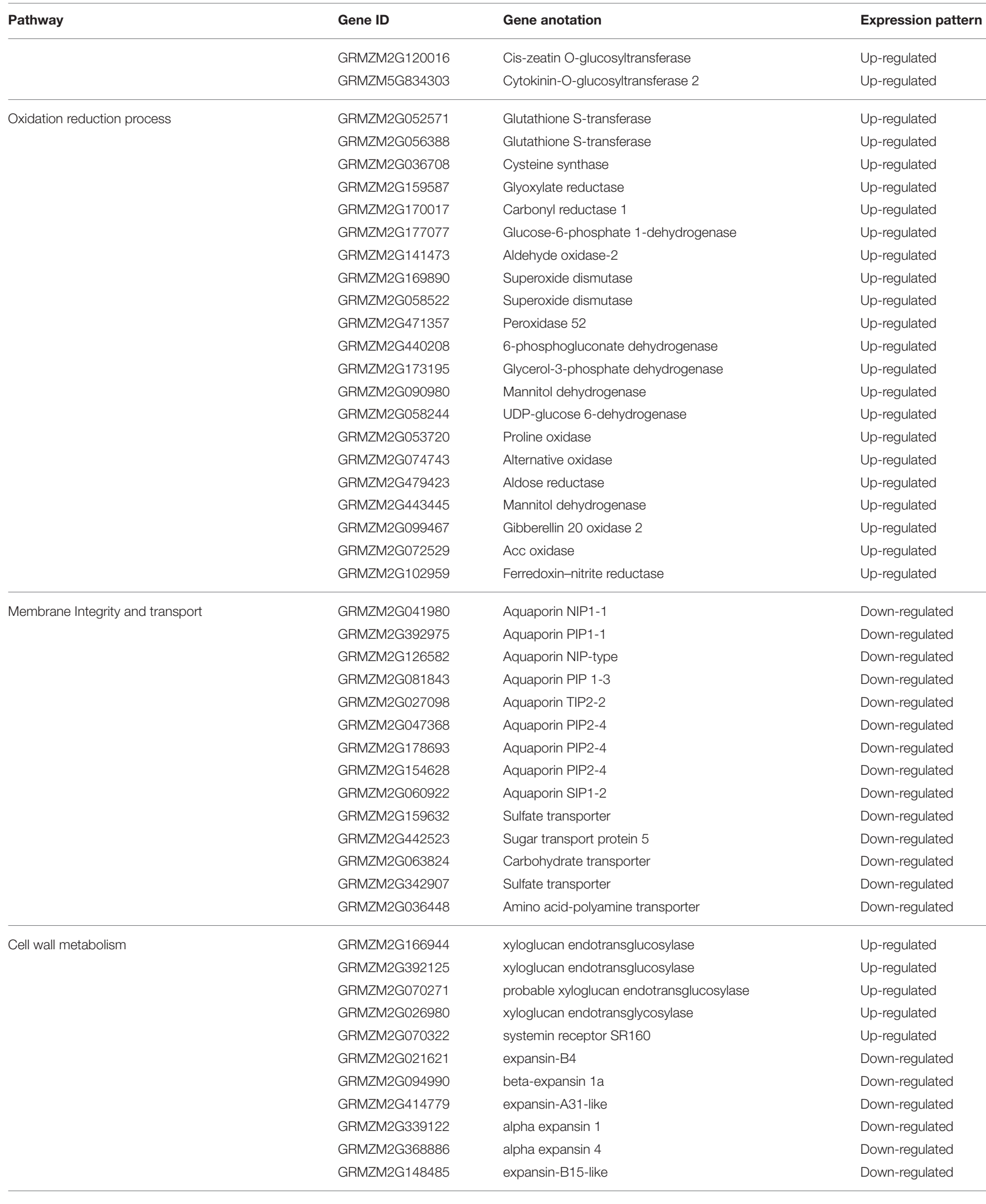


TABLE 3 | Continued

\begin{tabular}{|c|c|c|c|}
\hline Pathway & Gene ID & Gene anotation & Expression pattern \\
\hline & GRMZM2G082520 & beta-expansin 1a & Down-regulated \\
\hline & GRMZM2G013002 & beta expansin 8 & Down-regulated \\
\hline & GRMZM2G342246 & beta-expansin 7 & Down-regulated \\
\hline & GRMZM2G021427 & expansin-B3-like & Down-regulated \\
\hline & GRMZM2G025231 & cellulose synthase 7 & Down-regulated \\
\hline & GRMZM2G178025 & endoglucanase 12-like & Down-regulated \\
\hline & GRMZM2G453565 & endoglucanase 2-like & Down-regulated \\
\hline & GRMZM2G147687 & exoglucanase 1 & Down-regulated \\
\hline & GRMZM2G141911 & endoglucanase 4-like & Down-regulated \\
\hline & GRMZM2G131912 & pectate lyase 8 & Down-regulated \\
\hline & GRMZM2G091191 & brassinosteroid-regulated protein BRU1-like & Down-regulated \\
\hline
\end{tabular}

Conflict of Interest Statement: The authors declare that the research was conducted in the absence of any commercial or financial relationships that could be construed as a potential conflict of interest.
Copyright (c) 2017 Liu, Zhang, Fu, Wang, Wang and Liu. This is an open-access article distributed under the terms of the Creative Commons Attribution License (CC $B Y)$. The use, distribution or reproduction in other forums is permitted, provided the original author(s) or licensor are credited and that the original publication in this journal is cited, in accordance with accepted academic practice. No use, distribution or reproduction is permitted which does not comply with these terms. 